\begin{tabular}{|c|c|}
\hline Title & The Gauss maps of Demoulin surfaces with conformal coordinates In Memory of Professor Zhengguo Bai(1916-2015) \\
\hline Author(s) & Inoguchi, Jun-ichi; Kobay ashi, Shimpei \\
\hline Citation & $\begin{array}{l}\text { Science China mathematics, 64(7), 1479-1492 } \\
\text { https://doi.org/10.1007/s11425-020-1738-0 }\end{array}$ \\
\hline Issue Date & $2021-09-28$ \\
\hline Doc URL & http:/hdl.handle.net/2115/82779 \\
\hline Rights & $\begin{array}{l}\text { This is a post-peer-review, pre copy edit version of an article published in Science China mathematics. The final } \\
\text { authenticated version is avail able online at: } h t t p: / / d x . d o i . o r g / 10.1007 / 311425-020-1738-0 \text {. }\end{array}$ \\
\hline Type & article (author version) \\
\hline File Information & Sci. China Math.64 7_1479-1492.pdf \\
\hline
\end{tabular}

Instructions for use 


\title{
THE GAUSS MAPS OF DEMOULIN SURFACES WITH CONFORMAL COORDINATES
}

\author{
JUN-ICHI INOGUCHI AND SHIMPEI KOBAYASHI
}

In Memory of Professor Zhengguo Bai (1916-2015)

\begin{abstract}
Demoulin surfaces in real projective 3-space are investigated. Our result enable us to establish a generalized Weierstrass type representation for definite Demoulin surfaces by virtue of primitive maps into a certain semi-Riemannian 6 -symmetric space.
\end{abstract}

\section{INTRODUCTION}

Professor Zhengguo Bai have done great contributions in projective differential geometry. For example, he solved the so-called Fubini's problem [22] ( $c f$ [28]).

Projective differential geometry of surfaces is a treasure box of infinite dimensional integrable systems. For instance, harmonic maps of Riemann surfaces into complex projective space $\mathbb{C P}^{n}$ (the $\mathbb{C P}^{n}$-sigma models in particle physics) are typical examples of 2-dimensional integrable systems. One of the key clue of the study of harmonic maps into complex projective space is the use of harmonic sequences introduced by Chern and Wolfson [8]. It should be emphasized that the basic idea of harmonic sequence goes back to Laplace sequence in classical projective differential geometry, see [3].

From modern point of view, the Laplace sequence produces 2-dimensional Toda field equation of type $A_{\infty}$, see [9, 25. In particular, the periodic Laplace sequence produces 2-dimensional periodic Toda field equations. For example, Laplace sequences of period 2 produce sinh-Gordon equation. Titeica equation is obtained as Laplace sequence of period 3 , and it is a structure equation of affine spheres [11]. Laplace sequences of period 4 were studied by $\mathrm{Su}$ [26, 27]. Hu gave a Darboux matrix, that is, the simple type dressing for such a sequence [14].

This article addresses Laplace sequences of period 6. The Toda field equation derived from those sequences is a structure equation of Demoulin surfaces in real projective 3-space $\mathbb{R} \mathbb{P}^{3}$

Godeaux gave a method for studying projective surfaces through their Plüker images in real projective 5 -space $\mathbb{R P}^{5}$. His method relies on the consideration of the Laplace sequence associated with the Plüker image, called the Godeaux sequence. For a characterization of

2010 Mathematics Subject Classification. Primary 53A20, Secondary 53C43, 37K10.

Key words and phrases. Demoulin surface, Wilczynski frame, Gauss map.

The first named author is partially supported by JSPS Kakenhi Grant Number JP19K03461.

The second named author is partially supported by JSPS Kakenhi Grant Number JP18K03265. 
Demoulin surfaces in terms of Godeaux sequences, see [25, §4.8]. Bai [21] studied Godeaux sequences of quadrics.

In [15], the second named author considered two Gauss maps of surfaces in $\mathbb{R P}^{3}$ with indefinite projective metric and characterized projective minimal surfaces and Demoulin surfaces in terms of harmonicities of the Gauss maps. In this paper, we consider those surfaces with positive definite projective metric. This paper is organized as follows: After preparing prerequisite knowledge on projective surface theory in Sections 1 13 , we parametrize the space of all conformal 2-spheres in $\mathbb{R P}^{3}$ in Section 4 . We will show that the space of all conformal 2-spheres is realized as a semi-Riemannian symmetric space. The Gauss maps introduced in this paper take values in this symmetric space. In Section 5 , we introduce the first-order Gauss map for a surface in $\mathbb{R P}^{3}$ as a congruence of conformal 2-spheres which has the first-order contact to the surface. Definite Demoulin surfaces are characterized as surfaces with conformal first order Gauss map. In addition definite Demoulin surfaces and definite projective minimal coincidence surfaces are characterized by harmonicity of first order Gauss map. In the final section we will show that every definite Demoulin surface can be constructed by a primitive map into certain semi-Riemannian 6-symmetric space fibered over the semi-Riemannian symmetric space of all conformal 2-spheres.

Throughout this paper, we use the following abbreviation:

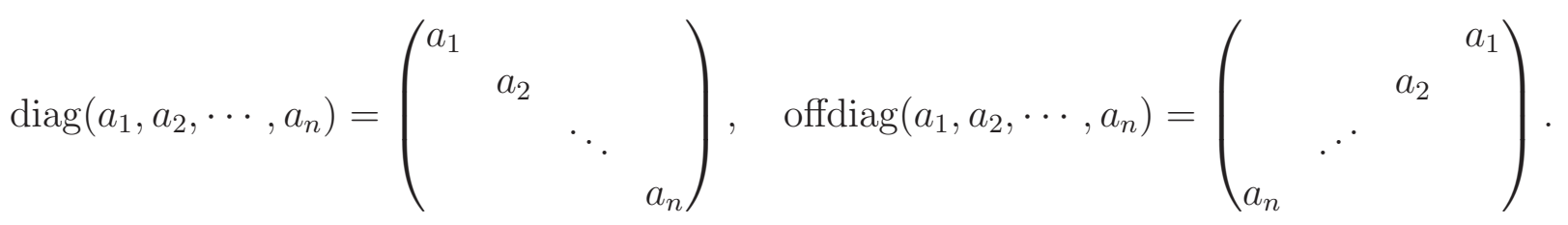

\section{Projective surface theory}

Let $\mathfrak{f}: M \rightarrow \mathbb{R P}^{3}$ be an immersed surface in the real projective 3 -space $\mathbb{R} \mathbb{P}^{3}$. Take a simply connected region $\mathbb{D} \subset M$ and homogeneous coordinate vector field $f=\left(f^{0}, f^{1}, f^{2}, f^{3}\right)$ : $\mathbb{D} \rightarrow \mathbb{R}^{4} \backslash\{\mathbf{0}\}$. Let $D$ be the natural affine connection on $\mathbb{R}^{4}$ and $\Omega$ a volume element so that $D \Omega=0$. Thus $\left(\mathbb{R}^{4}, D, \Omega\right)$ is an equiaffine 4 -space. One can take a vector field $\xi$ transversal to both $f$ and the radial vector field $\zeta=\sum_{i=0}^{3} x^{i} \partial / \partial x^{i}$. Then $\xi$ induces an affine connection $\nabla$ on $\mathbb{D}$ and symmetric tensor fields $h$ and $T$ via the Gauss formula:

$$
D_{X} f_{*} Y=f_{*}\left(\nabla_{X} Y\right)+h(X, Y) \xi+T(X, Y) \zeta, \quad X, Y \in \Gamma(T \mathbb{D}) .
$$

Moreover we have the following Weingarten formula:

$$
D_{X} \xi=-f_{*}(S X)+\tau(X) \xi+\rho(X) \zeta .
$$

The triplet $(\mathbb{D}, f, \xi)$ is a centroaffine surface (of codimension 2 ) in $\mathbb{R}^{4}$ in the sense of [19, 20]. We introduce an area element $\vartheta$ on $\mathbb{D}$ by

$$
\vartheta(X, Y)=\Omega\left(f_{*} X, f_{*} Y, \xi, \zeta\right) .
$$

The cubic form $C$ is defined by

$$
C=\nabla h+\tau \otimes h .
$$


The non-degeneracy of $h$ is independent of the choice of $\xi$. In addition, the conformal class $[h]$ of $h$ is independent of $\xi$. Thus the property " $h$ is positive definite" is well defined for $f$. Throughout this article, we assume that $h$ is positive definite.

When we take $\xi$ so that $\tau=0$, then $(\mathbb{D}, f, \xi)$ is said to be equiaffine. An equiaffine centroaffine immersion $f$ is said to be Blaschke if $\vartheta$ coincides with the area element of the metric $h$.

On the other hand Nomizu and Sasaki [19] showed that there exits a transversal vector field $\xi$ such that

$$
\operatorname{tr}_{h} T+\operatorname{tr} S=0
$$

Such a vector field is called a pre-normalized transversal vector field. In particular, prenormalized transversal vector field $\xi$ so that $(\mathbb{D}, f, \xi)$ is a Blaschke immersion is unique up to sign. In such a choice, the pair surface $(f, \xi)$ is called a pre-normalized Blaschke immersion.

Let us take another homogeneous coordinate vector field $\tilde{f}=\phi f$. Here $\phi$ is a smooth (nonzero) function. Then the connection $\tilde{\nabla}$ induced from $\tilde{f}$ is projectively equivalent to $\nabla$. The equiaffine property is preserved under the change $f$ by $\phi f$.

Let us denote by $\nabla^{h}$ the Levi-Civita connection of $h$. Then the scalar field $J=h(K, K) / 2$ is called the Fubini-Pick invariant of $f$. Here $K=\nabla-\nabla^{h}$. The Riemannian metric Jh is projectively invariant and called the projective metric of $\mathfrak{f}$. Although $C$ itself is not projective invariant, its conformal class is projective invariant (see [18]). When $(f, \xi)$ is pre-normalized Blaschke, the projective metric is given by $h(\nabla h, \nabla h) h / 8$.

For more details on centroaffine immersions and projective immersions, we refer to [19, 20].

\section{WILCZYNSKI FRAMES}

Let $\mathfrak{f}: M \rightarrow \mathbb{R P}^{3}$ be an immersed surface with positive definite projective metric. We regard $M$ as a Riemann surface with respect to the conformal structure $[J h]$ determined by the projective metric $J h$.

We take a simply connected complex coordinate region $\mathbb{D}$ with coordinate $z=x+i y$ on $\mathbb{D}$ and a lift $f=\left(f^{0}, f^{1}, f^{2}, f^{3}\right): \mathbb{D} \rightarrow \mathbb{R}^{4} \backslash\{\mathbf{0}\}$. Then the canonical system of $\mathfrak{f}$ is given by

$$
f_{z z}=b f_{\bar{z}}+p f, \quad f_{\bar{z} \bar{z}}=\bar{b} f_{z}+\bar{p} f
$$

for some smooth functions $b$ and $p$, see [25, p. 121]. Note that the subscripts $z$ and $\bar{z}$ denote the partial derivative of $z$ and $\bar{z}$, respectively:

$$
\frac{\partial}{\partial z}:=\frac{1}{2}\left(\frac{\partial}{\partial x}-\sqrt{-1} \frac{\partial}{\partial y}\right), \quad \frac{\partial}{\partial \bar{z}}:=\frac{1}{2}\left(\frac{\partial}{\partial x}+\sqrt{-1} \frac{\partial}{\partial y}\right) .
$$

Assume that $f^{0} \neq 0$, then $\mathfrak{f}$ is given by the inhomogeneous coordinate $\mathfrak{f}=\left(f^{1}, f^{2}, f^{3}\right) / f^{0}$. The canonical system is rewritten as

$$
\mathfrak{f}_{z z}=b \mathfrak{f}_{\bar{z}}-2\left(\log f^{0}\right)_{z} \mathfrak{f}_{z}, \quad \mathfrak{f}_{\bar{z} \bar{z}}=\bar{b} \mathfrak{f}_{z}-2\left(\log f^{0}\right)_{\bar{z}} \mathfrak{f}_{\bar{z}} .
$$


The integrability condition of the canonical system is (cf. [25, §2.3]):

$$
\begin{gathered}
p_{\bar{z}}=b \bar{b}_{z}+\frac{1}{2} b_{z} \bar{b}-\frac{1}{2} b_{\bar{z} \bar{z}}, \\
\operatorname{Im}\left(b_{\bar{z} \bar{z} \bar{z}}-b \bar{b}_{z \bar{z}}-2 b \bar{p}_{\bar{z}}-2 b_{\bar{z}} \bar{b}_{z}-4 b_{\bar{z}} \bar{p}\right)=0 .
\end{gathered}
$$

The Fubini-Pick invariant is given by $J=8|b|^{2}$ and hence the projective metric is $8|b|^{2} d z d \bar{z}$. The cubic form of $\mathfrak{f}$ is given by $C=-2\left(b d z^{3}+\bar{b} d \bar{z}^{3}\right)$ (see 24, p. 54, Definition, $\S 4.8]$ ). Note that when $f$ is pre-normalized Blashcke, then the projective metric is expressed as $2|b|^{2} d z d \bar{z}$.

Hereafter we assume that $b \neq 0$. Note that when $C=0, \mathfrak{f}$ is a part of a quadratic surface (Wilczynski [30], Pick 23]. See also [24, Theorem 4.4].)

The Wilczynski frame $F$ of $\mathfrak{f}$ is defined by

$$
F=\left(f, f_{1}, f_{2}, \eta\right)
$$

where

$$
f_{1}:=f_{z}-\frac{\bar{b}_{z}}{2 \bar{b}} f, \quad f_{2}:=f_{\bar{z}}-\frac{b_{\bar{z}}}{2 b} f, \quad \eta=f_{z \bar{z}}-\frac{\bar{b}_{z}}{2 \bar{b}} f_{\bar{z}}-\frac{b_{\bar{z}}}{2 b} f_{z}+\left(\frac{\left|b_{z}\right|^{2}}{4|b|^{2}}-\frac{|b|^{2}}{2}\right) f .
$$

Then a straightforward computation shows that the Wilczynski frame $F$ satisfies the following equations:

$$
F_{z}=F U \text { and } F_{\bar{z}}=F V
$$

where

$$
\begin{aligned}
U & =\left(\begin{array}{cccc}
\bar{b}_{z} /(2 \bar{b}) & P & k & b \bar{P} \\
1 & -\bar{b}_{z} /(2 \bar{b}) & 0 & k \\
0 & b & \bar{b}_{z} /(2 \bar{b}) & P \\
0 & 0 & 1 & -\bar{b}_{z} /(2 \bar{b})
\end{array}\right), \\
V & =\left(\begin{array}{cccc}
b_{\bar{z}} /(2 b) & \bar{k} & \bar{P} & \bar{b} P \\
0 & b_{\bar{z}} /(2 b) & \bar{b} & \bar{P} \\
1 & 0 & -b_{\bar{z}} /(2 b) & \bar{k} \\
0 & 1 & 0 & -b_{\bar{z}} /(2 b)
\end{array}\right) .
\end{aligned}
$$

Here we introduced functions $k$ and $P$ and $Q$ of as follows:

$$
\begin{gathered}
k=\frac{|b|^{2}-(\log b)_{z \bar{z}}}{2}, \\
P=p+\frac{b_{\bar{z}}}{2}-\frac{\bar{b}_{z z}}{2 \bar{b}}+\frac{\bar{b}_{z}^{2}}{4 \bar{b}^{2}} .
\end{gathered}
$$


The compatibility conditions of $(2.3)$ are

$$
\begin{gathered}
\bar{P}_{z}=k_{\bar{z}}+k \frac{b_{\bar{z}}}{b}, \\
\operatorname{Im}\left(\bar{b} P_{z}+2 \bar{b}_{z} P\right)=0 .
\end{gathered}
$$

These equations are nothing but the projective Gauss-Codazzi equations of a surface $\mathfrak{f}$. One can see that $P d z^{2}$ and $2 b^{2} \bar{P} d z^{4}$ are globally defined on $M$ and projectively invariant 13 .

Since both $U$ and $V$ are trace free, the Wilczynski frame $F$ takes values in $\mathrm{SL}_{4} \mathbb{C}$ up to initial condition. Moreover, if we choose at some base point $z_{*} \in \mathbb{D}$ and $F\left(z_{*}\right)=\mathrm{id}$, then the frame $F$ takes values in $\mathrm{SL}_{4} \mathbb{R}$ by conjugation of a simple complex matrix:

$$
\operatorname{Ad}(L) F \in \mathrm{SL}_{4} \mathbb{R}, \quad L=\frac{1}{\sqrt{2}}\left(\begin{array}{cccc}
\sqrt{2} & 0 & 0 & 0 \\
0 & \sqrt{-1} & -\sqrt{-1} & 0 \\
0 & 1 & 1 & 0 \\
0 & 0 & 0 & \sqrt{2}
\end{array}\right) .
$$

\section{Projective minimal surfaces and Definite Demoulin surfaces}

A surface $\mathfrak{f}: M \rightarrow \mathbb{R P}^{3}$ with positive definite projective metric is said to be a projective minimal surface if it is a critical point of the area functional of the projective metric (called the projective area functional): Then the projective minimality can be computed as in [29]:

$$
\bar{b} P_{z}+2 \bar{b}_{z} P=0 \text {. }
$$

where the functions $P$ is defined in (2.5) . It should be remarked that the projective minimality (3.1) implies the second equation (2.7) of the projective Gauss-Codazzi equations. There is a particular class of projective minimal surfaces with positive definite projective metric.

A surface with positive definite projective metric is said to be a definite Demoulin surface if it satisfies $P=0$. The Demoulin property is originated from Demoulin transformations of surfaces in $\mathbb{R P}^{3}$. For more details, we refer to [25].

\section{The PlüCKER QUADRIC AND THE SPACE OF CONFORMAL SPHERES}

4.1. The Plücker quadric. Take a volume element $\Omega$ on $\mathbb{R}^{4}$ parallel with respect to the natural affine connection $D$. Then we can introduce a scalar product $\langle\cdot, \cdot\rangle$ on $\wedge^{2} \mathbb{R}^{4}$ by

$$
\langle\alpha, \beta\rangle=\Omega(\alpha \wedge \beta), \quad \alpha, \beta \in \wedge^{2} \mathbb{R}^{4} .
$$

One can check that $\langle\cdot, \cdot\rangle$ is of signature $(3,3)$. In fact, let $\left\{e_{0}, e_{1}, e_{2}, e_{3}\right\}$ be the natural basis of $\mathbb{R}^{4}$. Denote by $\left\{e^{0}, e^{1}, e^{2}, e^{3}\right\}$ the dual basis of $\left\{e_{0}, e_{1}, e_{2}, e_{3}\right\}$. Then with respect to the volume element $\Omega=e^{0} \wedge e^{1} \wedge e^{2} \wedge e^{3}$, the basis $\left\{e_{0} \wedge e_{1}, e_{0} \wedge e_{2}, e_{0} \wedge e_{3}, e_{1} \wedge e_{2}, e_{3} \wedge e_{1}, e_{2} \wedge e_{3}\right\}$ of $\wedge^{2} \mathbb{R}^{4}$, the scalar product $\langle\cdot, \cdot\rangle$ is determined by the matrix offdiag $(1,1,1,1,1,1)$. The special linear group $\mathrm{SL}_{4} \mathbb{R}$ acts on $\wedge^{2} \mathbb{R}^{4}$ via the action:

$$
\mathrm{SL}_{4} \mathbb{R} \times \wedge^{2} \mathbb{R}^{4} \rightarrow \wedge^{2} \mathbb{R}^{4} ; \quad(g, v \wedge w) \longmapsto g v \wedge g w .
$$


One can see that this action is isometric with respect to $\langle\cdot, \cdot\rangle$. This fact implies the Lie group isomorphism $\mathrm{PSL}_{4} \mathbb{R} \cong \mathrm{SO}_{3,3}^{+}$. Here $\mathrm{SO}_{3,3}^{+}$denotes the identity component of the semi-orthogonal group $\mathrm{O}_{3,3}$.

Next we consider the Plücker embedding of the Grassmannian manifold $\mathrm{Gr}_{2}\left(\mathbb{R}^{4}\right)$ of 2planes in $\mathbb{R}^{4}$ into the projective 5 -space $\mathbb{R} \mathbb{P}^{5}=\mathbb{P}\left(\wedge^{2} \mathbb{R}^{4}\right)$. The Plücker coordinates of the 2-plane spanned by $\left(a^{0}, a^{1}, a^{2}, a^{3}\right)$ and $\left(b^{0}, b^{1}, b^{2}, b^{3}\right)$ is $\left[p_{01}: p_{02}: p_{03}: p_{23}: p_{31}: p_{12}\right]$, where

$$
p_{i j}=\operatorname{det}\left(\begin{array}{ll}
a^{i} & b^{i} \\
a^{j} & b^{j}
\end{array}\right) .
$$

The Plücker coordinates $\left[p_{01}: p_{02}: p_{03}: p_{23}: p_{31}: p_{12}\right]$ of $a \wedge b$ satisfies the quadratic Plücker relation:

$$
p_{01} p_{23}+p_{02} p_{31}+p_{03} p_{12}=0 .
$$

Thus the Plücker image of $\mathrm{Gr}_{2}\left(\mathbb{R}^{4}\right)$ is a projective variety (called the Plücker quadric) of $\mathbb{R P}^{5}$ determined by the equation (4.2). Moreover the Plücker relation means that $\left(p_{01}, p_{02}, p_{03}, p_{23}, p_{31}, p_{12}\right)$ is null with respect to $\langle\cdot, \cdot\rangle$. Namely the Plücker image of $\mathrm{Gr}_{2}\left(\mathbb{R}^{4}\right)$ is the projective light cone $\mathbb{P}(\mathcal{L})$ of $\wedge^{2} \mathbb{R}^{4}=\mathbb{R}^{3,3}$.

Now let us consider a line $\ell$ in $\mathbb{R P}^{3}$ connecting two points $a=\left[a^{0}: a^{1}: a^{2}: a^{3}\right]$ and $b=\left[b^{0}: b^{1}: b^{2}: b^{3}\right]$. The Plücker image of $\ell$ in $\mathbb{R} \mathbb{P}^{5}=\mathbb{P}\left(\wedge^{2} \mathbb{R}^{4}\right)$ is

$$
a \wedge b=\left[p_{01}: p_{02}: p_{03}: p_{23}: p_{31}: p_{12}\right]
$$

with (4.1). Hence the space $\mathcal{P}$ of lines in $\mathbb{R P}^{3}$ is identified with the Plücker quadric. This identification is called the Klein correspondence.

Remark 4.1. The conformal compactification of semi-Euclidean 4-space $\mathbb{R}^{2,2}$ of neutral signature is obtained as the projective light cone $\mathbb{P}(\mathcal{L}) \subset \mathbb{R P}^{5}$ equipped with the conformal structure induced from $\mathbb{R}^{3,3}$. The action of $\mathrm{PSL}_{4} \mathbb{R} \cong \mathrm{SO}_{3,3}^{+}$on $\mathbb{P}(\mathcal{L})$ is conformal. One can see that the Plücker quadric $\mathcal{P}=\mathbb{P}(\mathcal{L})$ is isomorphic to $\operatorname{Gr}_{2}\left(\mathbb{R}^{4}\right) \cong\left(\mathbb{S}^{2} \times \mathbb{S}^{2}\right) / \mathbb{Z}_{2}$ (equipped with the standard conformal structure of neutral signature) as a conformal manifold. Note that on $\mathbb{P}(\mathcal{L})$, there exits a complex structure compatible to the standard neutral metric. The standard neutral metric is neutral Kähler with respect to the complex structure. In particular, the Kähler form is regarded as a standard symplectic form on $\mathbb{P}(\mathcal{L})$. For more information on conformal geometry of $\mathbb{P}(\mathcal{L})$, see [17].

4.2. The space of conformal spheres. A quadric in $\mathbb{R P}^{3}$ is a surface of the form $\{[v] \in$ $\left.\mathbb{R P}^{3} \mid q(v, v)=0\right\}$, where $q$ is a scalar product of $\mathbb{R}^{4}$. For our purpose we choose a Lorentzian scalar product $q=\langle\cdot, \cdot\rangle$ on $\mathbb{R}^{4}$ and regarded it as a Minkowski 4 -space $\mathbb{R}^{1,3}$. Then the quadric is nothing but the conformal 2-sphere (Riemann sphere) in $\mathbb{R P}^{3}$. The space of conformal 2 -spheres in $\mathbb{R P}^{3}$ is parametrized as the space $\mathcal{Q}$ of $3 \times 3$ symmetric matrices with determinant one and signature $(1,3)$. In fact, the conformal 2 -sphere is given by the Lorentzian scalar product $q(u, v)=u Q v^{\mathrm{T}}$ with $Q \in \mathcal{Q}$.

The special linear group $\mathrm{SL}_{4} \mathbb{R}$ acts transitively on $\mathcal{Q}$ via the action $(g, Q) \longmapsto g Q g^{\mathrm{T}}$ with $g \in \mathrm{SL}_{4} \mathbb{R}$ and $Q \in \mathcal{Q}$. The stabilizer at 


$$
\hat{J}_{1}=\left(\begin{array}{llll}
0 & 0 & 0 & 1 \\
0 & 1 & 0 & 0 \\
0 & 0 & 1 & 0 \\
1 & 0 & 0 & 0
\end{array}\right),
$$

is given by $\hat{K}_{1}=\left\{a \in \mathrm{SL}_{4} \mathbb{R} \mid a \hat{J}_{1} a^{\mathrm{T}}=\hat{J}_{1}\right\}$, which is isomorphic to the identity component $\mathrm{SO}_{1,3}^{+}$of the semi-orthogonal group $\mathrm{O}_{1,3}^{+}$of signature $(1,3)$. Thus $\mathcal{Q}$ is isomorphic to the homogeneous space $\mathrm{SL}_{4} \mathbb{R} / \mathrm{SO}_{1,3}^{+} \cong \mathrm{SO}_{3,3}^{+} / \mathrm{SO}_{1,3}^{+}$.

We introduce a scalar product $\langle\cdot, \cdot\rangle$ at $Q \in \mathcal{Q}$ by

$$
\langle X, Y\rangle_{Q}=\operatorname{Tr}\left(Q^{-1} X Q^{-1} Y\right), \quad X, Y \in T_{Q} \mathcal{Q} .
$$

Note that at the origin of $\mathrm{SO}_{3,3}^{+} / \mathrm{SO}_{1,3}^{+}$, and $8\langle\cdot, \cdot\rangle$ is the Killing form of $\mathfrak{s l}_{4} \mathbb{R}$. This scalar product is invariant under the action of $\mathrm{SL}_{4} \mathbb{R}$. In fact,

$$
\left\langle g X g^{\mathrm{T}}, g Y g^{\mathrm{T}}\right\rangle_{g Q g^{\mathrm{T}}}=\operatorname{Tr}\left(\left(g Q g^{\mathrm{T}}\right)^{-1} g X g^{\mathrm{T}}\left(g Q g^{\mathrm{T}}\right)^{-1} g Y g^{\mathrm{T}}\right)=\langle X, Y\rangle_{Q} .
$$

Thus $\mathcal{Q}=\mathrm{SL}_{4} \mathbb{R} / \hat{K}_{1}$ is a semi-Riemannian symmetric space corresponding to the outer involution

$$
\hat{\tau}_{1}(X)=\hat{J}_{1}\left(X^{\mathrm{T}}\right)^{-1} \hat{J}_{1} .
$$

Remark 4.2. The space of lines in the Plücker quadric $\mathcal{P}$ is identified with the Grassmannian manifold of all null 2-planes in $\mathbb{R}^{3,3}$ :

$$
\mathcal{Z}=\left\{W \in \mathrm{Gr}_{2}\left(\mathbb{R}^{3,3}\right) \mid W \text { is a null 2-plane in } \mathbb{R}^{3,3}\right\} \cong \mathrm{SO}_{3,3}^{+} / \mathrm{SO}_{2,2}^{+} .
$$

For surfaces in $\mathbb{R P}^{3}$ with indefinite projective metric, two kinds of Gauss maps are considered in our previous work [15]. Those Gauss maps take value in the space of quadrics determined by scalar products of signature $(2,2)$ of $\mathbb{R}^{4}$. The space of all quadrics derived from such scalar products are identified with the semi-Riemannian symmetric space $\mathrm{SO}_{3,3}^{+} / \mathrm{SO}_{2,2}^{+}$.

\section{Demoulin surfaces and the FIRst order Gauss maps}

In this section, we define the first-order Gauss map for a surface in $\mathbb{R P}^{3}$.

5.1. First-order Gauss map. Let $\mathfrak{f}: M \rightarrow \mathbb{R}^{3}$ be a surface and $F$ the corresponding Wilczynski frame defined in (2.3) with a base point $z_{*} \in \mathbb{D}$ and $F\left(z_{*}\right)=$ id. Let $L$ be the matrix defined in (2.8) and $\hat{F}$ be the $\mathrm{SL}_{4} \mathbb{R}$ matrix such that

$$
\operatorname{Ad}(L) F=\hat{F} .
$$

We now define the first order Gauss map $g_{1}$ as follows:

$$
g_{1}=\hat{F} \hat{J}_{1} \hat{F}^{\mathrm{T}}=\operatorname{Ad}(L)\left(F J_{1} F^{\mathrm{T}}\right),
$$

where the matrix $\hat{J}_{1}$ is the one given by (4.3) and $J_{1}=\operatorname{offdiag}(1,1,1,1)$. Note that $\operatorname{Ad}(L) J_{1}=\hat{J}_{1}$. Therefore the map $g_{1}$ takes values in the space of conformal 2-spheres:

$$
g_{1}: M \rightarrow \mathcal{Q} \cong \mathrm{SL}_{4} \mathbb{R} / \hat{K}_{1}=\mathrm{SL}_{4} \mathbb{R} / \mathrm{SO}_{1,3}^{+} \text {. }
$$


This map $g_{1}$ is known to be a quadric which has the first order contact to the surface and it does not have the second order contact, see [16, Section 22].

We now characterize the Demoulin surface by the first-order Gauss map.

Proposition 5.1. The first-order Gauss map $g_{1}$ of a surface $\mathfrak{f}$ in $\mathbb{R P}^{3}$ with positive definite projective metric is conformal if and only if $\mathfrak{f}$ is a definite Demoulin surface.

Proof. A direct computation shows that

$$
\partial_{z} g_{1}=2(L F)\left(\begin{array}{cccc}
b \bar{P} & k & P & 0 \\
k & 0 & 0 & 1 \\
P & 0 & b & 0 \\
0 & 1 & 0 & 0
\end{array}\right)(L F)^{\mathrm{T}}, \quad \partial_{\bar{z}} g_{1}=2(L F)\left(\begin{array}{cccc}
\bar{b} P & \bar{P} & \bar{k} & 0 \\
\bar{P} & \bar{b} & 0 & 0 \\
\bar{k} & 0 & 0 & 1 \\
0 & 0 & 1 & 0
\end{array}\right)(L F)^{\mathrm{T}} .
$$

Thus

$$
\left\langle\partial_{z} g_{1}, \partial_{z} g_{1}\right\rangle=16 P,\left\langle\partial_{\bar{z}} g_{1}, \partial_{\bar{z}} g_{1}\right\rangle=16 \bar{P} \text { and }\left\langle\partial_{z} g_{1}, \partial_{\bar{z}} g_{1}\right\rangle=\left\langle\partial_{\bar{z}} g_{1}, \partial_{z} g_{1}\right\rangle=8(k+\bar{k})+4|b|^{2} \text {. }
$$

Since the coordinates $(z, \bar{z})$ are null for the conformal structure induced by $\mathfrak{f}$, the first-order Gauss map $g_{1}$ is conformal if and only if $P=0$.

\subsection{Demoulin surfaces and projective minimal coincidence surfaces. We set}

$$
G=\operatorname{Ad}\left(L^{-1}\right) \mathrm{SL}_{4} \mathbb{R}=\left\{L^{-1} X L \mid X \in \mathrm{SL}_{4} \mathbb{R}\right\} \subset \mathrm{SL}_{4} \mathbb{C},
$$

where $L$ is defined in (2.8). The closed subgroup $G$ is a real form of $\mathrm{SL}_{4} \mathbb{C}$ which is isomorphic to $\mathrm{SL}_{4} \mathbb{R}$. The space $\mathcal{Q}$ of conformal 2 -spheres is isomorphic to $G / K_{1}$, where $K_{1}$ is

$$
K_{1}=\left\{a \in G \mid a J_{1} a^{\mathrm{T}}=J_{1}\right\} .
$$

Let $\tau_{1}$ be the outer involution on the $G$ associated to $G / K_{1}$ given by

$$
\tau_{1}(a)=J_{1}\left(a^{\mathrm{T}}\right)^{-1} J_{1}, \quad a \in G .
$$

By abuse of notation, we denote the differential of $\tau_{1}$ by the same letter $\tau_{1}$ :

$$
\tau_{1}(X)=-J_{1} X^{\mathrm{T}} J_{1}, \quad X \in \mathfrak{g} .
$$

Let us consider the eigenspace decomposition of $\mathfrak{g}$ with respect to $\tau_{1}$, that is, $\mathfrak{g}=\mathfrak{k}_{1} \oplus \mathfrak{p}_{1}$, where $\mathfrak{k}_{1}$ is the $(+1)$-eigenspace and $\mathfrak{p}_{1}$ is the $(-1)$-eigenspace as follows:

$$
\mathfrak{k}_{1}=\left\{\left(\begin{array}{cccc}
a_{11} & a_{12} & a_{13} & 0 \\
a_{21} & a_{22} & 0 & -a_{13} \\
a_{31} & 0 & -a_{22} & -a_{12} \\
0 & -a_{31} & -a_{21} & -a_{11}
\end{array}\right) \in \mathfrak{g}\right\}, \quad \mathfrak{p}_{1}=\left\{\left(\begin{array}{cccc}
a_{11} & a_{12} & a_{13} & a_{14} \\
a_{21} & -a_{11} & a_{23} & a_{13} \\
a_{31} & a_{32} & -a_{11} & a_{12} \\
a_{41} & a_{31} & a_{21} & a_{11}
\end{array}\right) \in \mathfrak{g}\right\} .
$$

We decompose the Maurer-Cartan form according to this decomposition $\alpha=F^{-1} d F=$ $U d z+V d \bar{z}$ along the Lie algebra decomposition $\mathfrak{g}=\mathfrak{k}_{1} \oplus \mathfrak{p}_{1}$. First we decompose $U$ and $V$ as

$$
U=U_{\mathfrak{k}_{1}}+U_{\mathfrak{p}_{1}}, \quad V=V_{\mathfrak{k}_{1}}+V_{\mathfrak{p}_{1}}, \quad U_{\mathfrak{k}_{1}}, V_{\mathfrak{k}_{1}} \in \mathfrak{k}_{1}, \quad U_{\mathfrak{p}_{1}}, V_{\mathfrak{p}_{1}} \in \mathfrak{p}_{1} .
$$

Next, set $\alpha_{\mathfrak{k}_{1}}=U_{\mathfrak{k}_{1}} d z+V_{\mathfrak{k}_{1}} d \bar{z}$ and $\alpha_{\mathfrak{p}_{1}}=U_{\mathfrak{p}_{1}} d z+V_{\mathfrak{p}_{1}} d \bar{z}$, then we obtain the expression 


$$
\alpha=\alpha_{\mathfrak{k}_{1}}+\alpha_{\mathfrak{p}_{1}}=U_{\mathfrak{k}_{1}} d z+V_{\mathfrak{k}_{1}} d \bar{z}+U_{\mathfrak{p}_{1}} d z+V_{\mathfrak{p}_{1}} d \bar{z}
$$

where $U=U_{\mathfrak{k}_{1}}+U_{\mathfrak{p}_{1}}$ and $V=V_{\mathfrak{k}_{1}}+V_{\mathfrak{p}_{1}}$. Let us insert the spectral parameter $\lambda \in \mathbb{S}^{1}$ into $U$ and $V$ as follows:

$$
U^{\lambda}=U_{\mathfrak{k}_{1}}+\lambda^{-1} U_{\mathfrak{p}_{1}} \text { and } V^{\lambda}=V_{\mathfrak{k}_{1}}+\lambda V_{\mathfrak{p}_{1}} .
$$

Then a $\mathbb{S}^{1}$-family of 1 -forms $\alpha_{\lambda}$ is defined as follows:

$$
\alpha^{\lambda}=\alpha_{\mathfrak{k}_{1}}+\lambda^{-1} \alpha_{\mathfrak{p}_{1}}^{\prime}+\lambda \alpha_{\mathfrak{p}_{1}}^{\prime \prime}=U^{\lambda} d z+V^{\lambda} d \bar{z}
$$

Using the matrices $U^{\lambda}$ and $V^{\lambda}$, they are explicitly given as follows:

$$
U^{\lambda}=\left(\begin{array}{cccc}
\bar{b}_{z} /(2 \bar{b}) & \lambda^{-1} P & \lambda^{-1} k & \lambda^{-1} b \bar{P} \\
\lambda^{-1} & -\bar{b}_{z} /(2 \bar{b}) & 0 & \lambda^{-1} k \\
0 & \lambda^{-1} b & \bar{b}_{z} /(2 \bar{b}) & \lambda^{-1} P \\
0 & 0 & \lambda^{-1} & -\bar{b}_{z} /(2 \bar{b})
\end{array}\right), \quad V^{\lambda}=\left(\begin{array}{cccc}
b_{\bar{z}} /(2 b) & \lambda \bar{k} & \lambda \bar{P} & \lambda \bar{b} \bar{P} \\
0 & b_{\bar{z}} /(2 b) & \lambda c & \lambda Q \\
\lambda & 0 & -b_{\bar{z}} /(2 b) & \lambda \bar{k} \\
0 & \lambda & 0 & -b_{\bar{z}} /(2 b)
\end{array}\right) .
$$

After these preparation, we obtain the following theorem.

Theorem 5.2. Let $\mathfrak{f}$ be a surface in $\mathbb{R P}^{3}$ with positive definite projective metric and $g_{1}$ the first-order Gauss map defined in (5.2). Moreover, let $\left\{\alpha^{\lambda}\right\}_{\lambda \in \mathbb{S}^{1}}$ be a family of 1-forms defined in (5.4). Then the following three properties are mutually equivalent:

1. The surface $\mathfrak{f}$ is a definite Demoulin surface or a projective minimal coincidence surface.

2. The first-order Gauss map $g_{1}$ is a harmonic map into $\mathcal{Q}$.

3. $\left\{d+\alpha^{\lambda}\right\}_{\lambda \in \mathbb{S}^{1}}$ is a family of flat connections on $\mathbb{D} \times G$.

Proof. Let us first compute the flatness

$$
d \alpha^{\lambda}+\frac{1}{2}\left[\alpha^{\lambda} \wedge \alpha^{\lambda}\right]=0, \quad \lambda \in \mathbb{S}^{1}
$$

for the connection $d+\alpha^{\lambda}$ on $\mathbb{D} \times G$. A straightforward computation shows that $d \alpha^{\lambda}+$ $\frac{1}{2}\left[\alpha^{\lambda} \wedge \alpha^{\lambda}\right]=0$ holds for all $\lambda \in \mathbb{S}^{1}$ if and only if

$$
P_{\bar{z}}=0, \quad k_{\bar{z}}+k \frac{b_{\bar{z}}}{b}=0, \quad \bar{b} P_{z}+2 \bar{b}_{z} P=0 .
$$

On can see that this system implies the projective Gauss-Codazzi equations (2.6) -(2.7). In particular, the third equation is nothing but the projective minimality equation (3.1).

Every definite Demoulin surface clearly satisfies the above flatness condition (zero curvature equations) since $P=0$.

Assume that $P \neq 0$. The first equation of (5.5) means that $P d z^{2}$ is a holomorphic differential. From the third equation together with the holomorphicity of $P$, one can deduce that $(\log \bar{b})_{z}$ is holomorphic. Hence $(\log b / \bar{b})_{z \bar{z}}=0$. Via the holomorphic coordinate change of $z$ preserving the form of canonical system, we can assume that $b=\bar{b}$, i.e., $b$ is real 1 .

\footnotetext{
${ }^{1}$ The transformation rule of $b$ under the conformal change of coordinates $w(z)$ is given by $\tilde{b}=\left(\bar{w}_{\bar{z}} / w_{z}^{2}\right) b$, and thus $\tilde{b}=\overline{\tilde{b}}$ can be achieved by a suitable choice of the function $w(z)$ under the condition $(\log b / \bar{b})_{z \bar{z}}=0$, see [13, Section 3].
} 
Then (2.4) implies that $2 k=b^{2}$. By using the second equation of (5.5), $b$ is constant and $k$ is a real constant. By using the third equation again, we get $P$ is constant. This implies that $P=p$ is a non-zero constant. After these reparametrization, the canonical system is rewritten as

$$
f_{z z}=b f_{\bar{z}}+p f, \quad f_{\bar{z} \bar{z}}=b f_{z}+p f .
$$

A surface satisfying the above equation is a special case of the coincidence surface, [25, Example 2.19]. In fact, it is easy to see that the surface is a projective minimal coincidence surface. Thus the equivalence of the claim 1 and claim 3 follows.

The equivalence of the claims 2 and 3 follows from Proposition A.2, since the $\mathbb{S}^{1}$-family of 1 -forms $\alpha^{\lambda}$ is given by the involution $\tau_{1}$ and it defines the semi-Riemannian symmetric space $\mathcal{Q}=\mathrm{SL}_{4} \mathbb{R} / K_{1}$.

Corollary 5.3. Retaining the assumptions in Theorem 5.2, the following are equivalent:

1. The surface $\mathfrak{f}$ is a definite Demoulin surface.

2. The first-order Gauss map $g_{1}$ is a conformal harmonic map into $\mathcal{Q}$.

Proof. From Proposition [5.1, it is easy to see that the first-order Gauss map is conformal if and only if it satisfies that $P=0$, that is, the surface is a definite Demoulin surface. Moreover, from Theorem 5.2 the Gauss map of the Demoulin surface is harmonic.

This corollary implies that if $\mathfrak{f}$ is a definite Demoulin surface or a projective minimal coincidence surface, then there exists a $\mathbb{S}^{1}$-parameter family of smooth map $F_{\lambda}: \mathbb{D} \times \mathbb{S}^{1} \rightarrow G$ which is a solution to

$$
\left(F_{\lambda}\right)^{-1} d F_{\lambda}=\alpha^{\lambda}
$$

under initial condition $F_{\lambda}\left(z_{*}\right)=\mathrm{id}$. One can see that $F_{\lambda}$ is regarded as a smooth map of $\mathbb{D}$ into the following twisted loop group

$$
\Lambda G_{\tau_{1}}=\left\{g: \mathbb{S}^{1} \rightarrow G \mid \tau_{1} g(\lambda)=g(-\lambda)\right\}
$$

of $G$. The $\Lambda G_{\tau_{1}}$-valued map $F_{\lambda}$ is referred as to the extended Wilczynski frame of a definite Demoulin surface.

Precisely speaking, the extended Wilczynski frame $F_{\lambda}$ is not the Wilczynski frame of a Demoulin surface or a projective minimal coincidence surface except for $\lambda=1$. By conjugating $F_{\lambda}$ by $D F_{\lambda} D^{-1}$ with $D=\operatorname{diag}\left(1, \lambda, \lambda^{-1}, 1\right)$, the frames $D F_{\lambda} D^{-1}$ give a family of Wilczynski frames for Demoulin surfaces or projective minimal coincidence surfaces. The corresponding Demoulin surfaces or projective minimal coincidence surfaces have the same projective metric $8|b|^{2} d z d \bar{z}$ but the different conformal classes of cubic forms $\lambda^{-3} b d z^{3}$. Moreover, the functions $P$ changes as $\lambda^{-2} P$.

\section{Primitive Lifts}

We now show that the extended Wilczynski frame for a Demoulin surface has an additional order three cyclic symmetry. Let $\sigma$ be an order three automorphism on the complexification $\mathrm{SL}_{4} \mathbb{C}$ of $G$ as follows:

$$
\sigma X=\operatorname{Ad}(E) X, \quad X \in \mathrm{SL}_{4} \mathbb{C},
$$


where $E=\operatorname{diag}\left(1, \epsilon^{2}, \epsilon, 1\right)$ with $\epsilon=e^{2 \pi \sqrt{-1} / 3}$. It should be emphasized that $\sigma$ preserves the real form $G$. Thus $\sigma$ is regarded as an automorphisms of $G$.

Next, one can check that $F_{\lambda}$ satisfies the symmetry $\sigma\left(F_{\lambda}\right)=F_{\epsilon \lambda}$, since $U^{\lambda}$ and $V^{\lambda}$ satisfy the same symmetry. It is also easy to see that $\tau_{1}$ and $\sigma$ commute, and $\kappa=\tau_{1} \circ \sigma$ defines an automorphism of order six. We obtain a regular semi-Riemannian 6-symmetric space $G / K$ (see Appendix A.1), where

$$
K=\left\{\operatorname{diag}\left(k_{1}, k_{2}, k_{2}^{-1}, k_{1}^{-1}\right) \mid k_{1} \in \mathbb{R}^{\times}, k_{2} \in \mathbb{S}^{1}\right\} \cong \mathrm{SO}_{1,1} \times \mathrm{SO}_{2} .
$$

Note that $G / K$ is identified with $\left\{g J g^{\mathrm{T}} \mid g \in G\right\}$, where $J=E J_{1}$. There is a homogeneous projection

$$
\pi: G / K \rightarrow G / K_{1} ; g K \longmapsto g K_{1} .
$$

The extended Wilczynski frame $F_{\lambda}$ satisfies the symmetry

$$
\kappa\left(F_{\lambda}\right)=F_{-\epsilon \lambda} .
$$

Note that $-\epsilon$ is the 6 th root of unity. From the above argument, it is easy to see that the extended Wilczynski frame $F_{\lambda}=F(\lambda)$ for a Demoulin surface is an element of the twisted loop group of $G$ :

$$
\Lambda G_{\kappa}=\left\{g: \mathbb{S}^{1} \rightarrow G \mid \kappa g(\lambda)=g(-\epsilon \lambda)\right\} .
$$

Theorem 6.1. The first-order Gauss map of a Demoulin surface, which is conformal harmonic into $\mathcal{Q}=G / K_{1}$, can be obtained by the homogeneous projection of a primitive map into the regular semi-Riemannian 6 -symmetric space $G / K \cong \mathrm{SL}_{4} \mathbb{R} / \mathrm{SO}_{1,1} \times \mathrm{SO}_{2}$.

Proof. The 0th-eigenspace $\mathfrak{g}_{0}^{\mathbb{C}}$ and \pm 1 st-eigenspaces $\mathfrak{g}_{ \pm 1}^{\mathbb{C}}$ of the derivative of the order six automorphism $\kappa=\tau_{1} \circ \sigma$ are described as follows:

$$
\mathfrak{g}_{0}^{\mathbb{C}}=\left\{\operatorname{diag}\left(a_{11}, a_{22},-a_{22},-a_{11}\right) \mid a_{11} \in \mathbb{R}, a_{22} \in \mathbb{C}\right\},
$$

and

$$
\mathfrak{g}_{-1}^{\mathbb{C}}=\left\{\left(\begin{array}{cccc}
0 & 0 & a_{13} & 0 \\
a_{21} & 0 & 0 & a_{13} \\
0 & a_{32} & 0 & 0 \\
0 & 0 & a_{21} & 0
\end{array}\right) \mid a_{i j} \in \mathbb{C}\right\}, \quad \mathfrak{g}_{1}^{\mathbb{C}}=\left\{\left(\begin{array}{cccc}
0 & a_{12} & 0 & 0 \\
0 & 0 & a_{23} & 0 \\
a_{31} & 0 & 0 & a_{12} \\
0 & a_{31} & 0 & 0
\end{array}\right) \mid a_{i j} \in \mathbb{C}\right\} .
$$

From the matrices $U^{\lambda}$ and $V^{\lambda}$ in (5.4) with $P=0$, we see that the condition in Definition A.1 of primitive map is satisfied. The stabilizer of $\kappa$ is the closed subgroup $K$ given by (6.1). Therefore there is a primitive map $g=F J F^{\mathrm{T}} J=E J_{1}$ into the 6 -symmetric space $G / K$ such that $\pi \circ g=\operatorname{Ad}\left(L^{-1}\right) g_{1}$. Since $\operatorname{Ad}\left(L^{-1}\right): \mathrm{SL}_{4} \mathbb{R} / \hat{K}_{1} \rightarrow G / K_{1}$ is an isometry, $g_{1}=\operatorname{Ad}(L)(\pi \circ g)$ is harmonic.

This theorem enable us to establish a generalized Weierstrass type representation for definite Demoulin surfaces by virtue of primitive maps into the semi-Riemannian 6-symmetric space $G / K$, see [12].

Remark 6.2. Corresponding result theorem for indefinite Demoulin surfaces was obtained by the second named author in the preprint version of [15]. 


\section{Appendix A. PRimitive haRmonic MAPS}

A.1. Homogeneous geometry. Let $G$ be a semi-simple real Lie group with automorphism $\tau$ of order $k \geq 2$. We consider a reductive homogenous space $G / K$ equipped with a $G$-invariant semi-Riemannian metric satisfying the following three conditions:

- The closed subgroup $H$ satisfies $G_{\tau}^{\circ} \subset K \subset G_{\tau}$. Here $G_{\tau}$ is the Lie subgroup of all fixed points of $\tau$ and $G_{\tau}^{\circ}$ the identity component of it.

- The $G$-invariant semi-Riemannian metric is derived from (a constant multiple of) the Killing form of $G$.

- The Lie algebra $\mathfrak{k}$ of $K$ is non-degenerate with respect to the induced scalar product.

The resulting homogeneous semi-Riemannian space $G / K$ is called a regular semi-Riemannian $k$-symmetric space. Note that a regular semi-Riemannian 2 -symmetric spaces is just a semiRiemannian symmetric space. Since $\mathfrak{k}$ is non-degenerate, the orthogonal complement $\mathfrak{p}$ of $\mathfrak{k}$ is non-degenerate and can be identified with the tangent space of $G / K$ at the origin $o=K$. The Lie algebra $\mathfrak{g}$ is decomposed into the direct sum:

$$
\mathfrak{g}=\mathfrak{k} \oplus \mathfrak{p} .
$$

of linear subspaces.

We denote the induced Lie algebra automorphism of $\mathfrak{g}$ by the same letter $\tau$. Now we have the eigenspace decomposition of the complexified Lie algebra $\mathfrak{g}^{\mathbb{C}}$;

$$
\mathfrak{g}^{\mathbb{C}}=\sum_{j \in \mathbb{Z}_{k}} \mathfrak{g}_{j}^{\mathbb{C}}
$$

where $\mathfrak{g}_{j}^{\mathbb{C}}$ is the eigenspace of $\tau$ with eigenvalue $\omega^{j}$. Here $\omega$ is the (primitive) $k$-th root of unity. In particular, $\mathfrak{g}_{0}^{\mathbb{C}}=\mathfrak{k}^{\mathbb{C}}$ and $\mathfrak{g}_{-1}^{\mathbb{C}}=\overline{\mathfrak{g}_{1}^{\mathbb{C}}}$. Let us define a subbundle $\left[\mathfrak{g}_{j}^{\mathbb{C}}\right]$ of $G / K \times \mathfrak{g}$ by

$$
\left[\mathfrak{g}_{j}^{\mathbb{C}}\right]_{g \cdot o}=\operatorname{Ad}(g) \mathfrak{g}_{j}^{\mathbb{C}} .
$$

Then the complexified tangent bundle $T^{\mathbb{C}} G / K$ is expressed as

$$
T^{\mathbb{C}} G / K=\sum_{j \in \mathbb{Z}_{k}, j \neq 0}\left[\mathfrak{g}_{j}^{\mathbb{C}}\right] .
$$

A.2. Primitive maps. A smooth map $\psi: \Sigma \rightarrow N$ of a Riemann surface $\Sigma$ into a semiRiemannian manifold $N$ is said to be a harmonic map if its tension field $\operatorname{tr}(\nabla d \psi)$ vanishes.

For smooth maps into regular semi-Riemannian $k$-symmetric spaces with $k>2$, the notion of primitive map was introduced by Burstall-Pedit [5] (see also Bolton-Pedit-Woodward [2]).

Definition A.1. Let $\psi: \Sigma \rightarrow G / K$ be a smooth map of a Riemann surface $\Sigma$ into a regular semi-Riemannian $k$-symmetric space with $k>2$. Then $\psi$ is said to be a primitive map if $\mathrm{d} \psi\left(T^{\prime} \Sigma\right) \subset\left[\mathfrak{g}_{-1}^{\mathbb{C}}\right]$. Here $T^{\prime} \Sigma$ denotes the (1,0)-tangent bundle of $\Sigma$.

Black [1] showed that primitive maps are equi-harmonic, that is, harmonic with respect to suitable invariant metrics on $G / K$ (see also [5]). In addition primitive maps well behave with respect to homogeneous projections [5, Theorem 3.7].

Theorem A.1. Let $H$ be a closed subgroup of $G$ satisfying 
- $K \subset H$.

- The Lie algebra $\mathfrak{h}$ of $H$ is non-degenerate.

- The decomposition $\mathfrak{g}=\mathfrak{h} \oplus \mathfrak{q}$ is reductive and stable under $\tau$. Here $\mathfrak{q}$ is the orthogonal complement of $\mathfrak{h}$.

Denote by $\pi_{H}: G / K \rightarrow G / H$ be the homogenous projection. Then for any primitive map $\psi, \pi_{H} \circ \psi$ is a harmonic map into $G / H$.

Note that when $k=2,\left[\mathfrak{g}_{-1}^{\mathbb{C}}\right]=T^{\mathbb{C}} G / K$ and the primitivity condition is vacuous. On the other hand when $k>2$, every primitive map is harmonic with respect to the Killing metric. To provide a unified description, we recall the following terminology from [6].

Definition A.2. A smooth map $\psi: \Sigma \rightarrow G / K$ into a regular semi-Riemannian $k$ symmetric space is said to be a primitive harmonic map if it is primitive for $k>2$ and harmonic if $k=2$.

Now let $\psi: \mathbb{D} \rightarrow G / H$ be a smooth map from a simply connected Riemann surface $\mathbb{D}$ into a regular semi-Riemannian $k$-symmetric space $G / K$ with $k \geq 2$. Take a frame $\Psi: \mathbb{D} \rightarrow G$ of $\psi$ and put $\alpha:=\Psi^{-1} d \Psi$. Then we have the identity (Maurer-Cartan equation):

$$
d \alpha+\frac{1}{2}[\alpha \wedge \alpha]=0
$$

Decompose $\alpha$ along the Lie algebra decomposition $\mathfrak{g}=\mathfrak{k} \oplus \mathfrak{p}$ as

$$
\alpha=\alpha_{\mathfrak{k}}+\alpha_{\mathfrak{p}}, \quad \alpha_{\mathfrak{k}} \in \mathfrak{k}, \quad \alpha_{\mathfrak{p}} \in \mathfrak{p} .
$$

We decompose $\alpha_{\mathfrak{p}}$ with respect to the conformal structure of $\mathbb{D}$ as

$$
\alpha_{\mathfrak{p}}=\alpha_{\mathfrak{p}}^{\prime}+\alpha_{\mathfrak{p}}^{\prime \prime}
$$

Here $\alpha_{\mathfrak{p}}^{\prime}$ and $\alpha_{\mathfrak{p}}^{\prime \prime}$ are the $(1,0)$ and $(0,1)$-part of $\alpha_{\mathfrak{p}}$, respectively. Since $G$ is a real Lie group, $\alpha_{\mathfrak{p}}^{\prime \prime}$ is the conjugate of $\alpha_{\mathfrak{p}}^{\prime}$.

Now let us assume that $\psi$ is a primitive harmonic map, then $\alpha_{\mathfrak{p}}^{\prime}$ is $\left[\mathfrak{g}_{-1}^{\mathbb{C}}\right]$-valued and $\alpha_{\mathfrak{p}}^{\prime \prime}$ is $\left[\mathfrak{g}_{1}^{\mathbb{C}}\right]$-valued, respectively. Hence the decomposition of $\alpha$ is rewritten as

$$
\alpha=\alpha_{-1}^{\prime}+\alpha_{0}+\alpha_{1}^{\prime} .
$$

Now let us introduce a spectral parameter $\lambda \in \mathbb{S}^{1}$ into $\alpha$ as

$$
\alpha^{\lambda}:=\alpha_{0}+\lambda^{-1} \alpha_{-1}^{\prime}+\lambda \alpha_{1}^{\prime \prime} .
$$

We arrive at the zero curvature representation of primitive harmonic maps:

Proposition A.2. Let $\mathbb{D}$ be a connected open subset of $\mathbb{C}$. Let $\psi: \mathbb{D} \rightarrow G / K$ be a primitive harmonic map. Then the loop of connections $d+\alpha^{\lambda}$ is flat for all $\lambda$, that is,

$$
d \alpha^{\lambda}+\frac{1}{2}\left[\alpha^{\lambda} \wedge \alpha^{\lambda}\right]=0
$$

for all $\lambda$. 
Conversely assume that $\mathbb{D}$ is simply connected. Let $\alpha^{\lambda}=\alpha_{0}+\lambda^{-1} \alpha_{-1}^{\prime}+\lambda \alpha_{1}^{\prime \prime}$ be an $\mathbb{S}^{1}$-family of $\mathfrak{g}$-valued one-forms which satisfies

$$
d \alpha^{\lambda}+\frac{1}{2}\left[\alpha^{\lambda} \wedge \alpha^{\lambda}\right]=0
$$

for all $\lambda \in \mathbb{S}^{1}$. Then there exists a one-parameter family of maps $\Psi_{\lambda}: \mathbb{D} \rightarrow G$ such that

$$
\Psi_{\lambda}^{-1} d \Psi_{\lambda}=\alpha^{\lambda},
$$

and

$$
\psi_{\lambda}=\Psi_{\lambda} \bmod K: \mathbb{D} \rightarrow G / K
$$

is primitive harmonic for all $\lambda$.

Appendix B. Projective minimal surfaces and the Conformal Gauss maps B.1. Conformal Gauss map. Let $\mathfrak{f}: M \rightarrow \mathbb{R}^{3}$ be a surface with Wilczynski frame $F$ as in subsection 5.1. We define a map $g_{2}$ by

$$
g_{2}=\hat{F} \hat{J}_{2} \hat{F}^{\mathrm{T}}=-\operatorname{Ad}(L)\left(F J_{2} F^{\mathrm{T}}\right), \quad \hat{J}_{2}=-L J_{2} L^{\mathrm{T}},
$$

where $J_{2}=\operatorname{offdiag}(1,-1,-1,1)$ ( $c f$. [24, $\left.\left.\S 4.1\right]\right)$. Analogous to the first-order Gauss map $g_{1}, g_{2}$ takes value in the space $\mathcal{Q}$ of conformal 2 -spheres in $\mathbb{R} \mathbb{P}^{3}$. More precisely, since the matrix $\hat{J}_{2}$ is of signature $(1,3)$, it is a point of $\mathcal{Q}$. Thus $\mathcal{Q}$ is realized as a homogeneous space $\mathrm{SL}_{4} \mathbb{R} / \hat{K}_{2}$, where $\hat{K}_{2}$ is the stabilizer at $\hat{J}_{2} \in \mathcal{Q}$ explicitly given by $\hat{K}_{2}=\{X \in$ $\left.\mathrm{SL}_{4} \mathbb{R} \mid X J_{2} X^{\mathrm{T}}=J_{2}\right\}$, which is also isomorphic to $\mathrm{SO}_{1,3}^{+}$. Thus the map $g_{2}$ takes value in $\mathrm{SL}_{4} \mathbb{R} / \hat{K}_{2}$ :

$$
g_{2}: M \rightarrow \mathcal{Q} \cong \mathrm{SL}_{4} \mathbb{R} / \hat{K}_{2}=\mathrm{SL}_{4} \mathbb{R} / \mathrm{SO}_{1,3}^{+} .
$$

This map $g_{2}$ is known to be a Lie quadric which has the second order contact to the surface, see [16, Section 18]. The map $g_{2}$ has been called the conformal Gauss map for a surface $\mathfrak{f}$ in $\mathbb{R P}^{3}$, see [29, 4]. In [18], the conformal Gauss map $g_{2}$ was called the projective Gauss map. In classical literature, $g_{2}$ was called the congruence of Lie quadrics.

Proposition B.1 (Theorem 3 in [4]). The conformal Gauss map $g_{2}$ is conformal map.

Proof. As in the proof of Proposition 5.1, a direct computation shows that

$$
\partial_{z} g_{2}=-2(L F) \operatorname{diag}(b \bar{P}, 0,-b, 0)(L F)^{\mathrm{T}} \text { and } \partial_{\bar{z}} g_{2}=-2(L F) \operatorname{diag}(\bar{b} P,-\bar{b}, 0,0)(L F)^{\mathrm{T}} .
$$

Thus

$$
\left\langle\partial_{z} g_{2}, \partial_{z} g_{2}\right\rangle=\left\langle\partial_{\bar{z}} g_{2}, \partial_{\bar{z}} g_{2}\right\rangle=0 \text { and }\left\langle\partial_{z} g_{2}, \partial_{\bar{z}} g_{2}\right\rangle=4|b|^{2} \neq 0 .
$$

Since the coordinates $(z, \bar{z})$ are null for the conformal structure induced by $\mathfrak{f}$, the conformal Gauss map $g_{2}$ is conformal.

Remark B.2. The Hodge star operator $\star$ on $\wedge^{2} \mathbb{R}^{1,3}$ is introduced by

$$
\langle a, b\rangle=\Omega(a \wedge \star b) .
$$


Since $\langle\cdot, \cdot\rangle$ is Lorentzian, $\star$ satisfies $\star^{2}=-1$. Thus the complexification $\left(\wedge^{2} \mathbb{R}^{1,3}\right)^{\mathbb{C}} \cong \wedge^{2} \mathbb{C}^{1,3}$ has the eigenspace decomposition

$$
\left(\wedge^{2} \mathbb{R}^{4}\right)^{\mathbb{C}}=\mathrm{S} \oplus \overline{\mathrm{S}}
$$

where $\mathrm{S}$ is the $\sqrt{-1}$-eigenspace of $\star$. In this way, a quadric $Q \in \mathcal{Q}$ corresponds to a complex linear subspace $\mathrm{S}$ of $\left(\wedge^{2} \mathbb{R}^{4}\right)^{\mathbb{C}}$. The correspondence $Q \longmapsto \mathrm{S}$ defines a smooth bijection from the space $\mathcal{Q}$ of conformal 2 -spheres in $\mathbb{R P}^{3}$ to the space

$$
\mathcal{G}_{2,0}^{3,3}=\left\{\mathrm{S} \subset\left(\mathbb{R}^{3,3}\right)^{\mathbb{C}} \mid \mathrm{S} \cap \mathrm{S}^{\perp}=\{0\}, \quad \overline{\mathrm{S}}=\mathrm{S}^{\perp}\right\} .
$$

Under this identification, $g_{2}$ is regarded as a smooth map into $\mathcal{G}_{2,0}^{3,3}$ in [4, p. 183], [7, p. 30].

B.2. Projective minimal surfaces and the conformal Gauss maps. The space $\mathcal{Q}$ of conformal 2-spheres in $\mathbb{R P}^{3}$ is isomorphic to the semi-Riemannian symmetric space $G / K_{2}$, where

$$
K_{2}=\left\{a \in G \mid a J_{2} a^{\mathrm{T}}=J_{2}\right\} .
$$

Let $\tau_{2}$ be the outer involution on $G$ associated to the symmetric space $G / K_{2}$ defined by:

$$
\tau_{2}(a)=J_{2}\left(a^{\mathrm{T}}\right)^{-1} J_{2}
$$

where $a \in G$. By abuse of notation, we denote the differential of $\tau_{2}$ by the same letter $\tau_{2}$ which is an outer involution on $\mathfrak{g}$ :

$$
\tau_{2}(X)=-J_{2} X^{\mathrm{T}} J_{2}
$$

where $X \in \mathfrak{g}$. Let us consider the eigenspace decomposition of $\mathfrak{g}$ with respect to $\tau_{2}$, that is, $\mathfrak{g}=\mathfrak{k}_{2} \oplus \mathfrak{p}_{2}$, where $\mathfrak{k}_{2}$ is the $(+1)$-eigenspace and $\mathfrak{p}_{2}$ is the $(-1)$-eigenspace as follows:

$$
\mathfrak{k}_{2}=\left\{\left(\begin{array}{cccc}
a_{11} & a_{12} & a_{13} & 0 \\
a_{21} & a_{22} & 0 & a_{13} \\
a_{31} & 0 & -a_{22} & a_{12} \\
0 & a_{31} & a_{21} & -a_{11}
\end{array}\right) \in \mathfrak{g}\right\}, \quad \mathfrak{p}_{2}=\left\{\left(\begin{array}{cccc}
a_{11} & a_{12} & a_{13} & a_{14} \\
a_{21} & -a_{11} & a_{23} & -a_{13} \\
a_{31} & a_{32} & -a_{11} & -a_{12} \\
a_{41} & -a_{31} & -a_{21} & a_{11}
\end{array}\right) \in \mathfrak{g}\right\}
$$

According to this decomposition $\mathfrak{g}=\mathfrak{k}_{2} \oplus \mathfrak{p}_{2}$, the Maurer-Cartan form $\alpha=F^{-1} d F=$ $U d z+V d \bar{z}$ can be decomposed into

$$
\alpha=\alpha_{\mathfrak{k}_{2}}+\alpha_{\mathfrak{p}_{2}}=U_{\mathfrak{k}_{2}} d z+V_{\mathfrak{k}_{2}} d \bar{z}+U_{\mathfrak{p}_{2}} d z+V_{\mathfrak{p}_{2}} d \bar{z}
$$

where $U=U_{\mathfrak{k}_{2}}+U_{\mathfrak{p}_{2}}$ and $V=V_{\mathfrak{k}_{2}}+V_{\mathfrak{p}_{2}}$. Let us insert the parameter $\lambda \in \mathbb{S}^{1}$ into $U$ and $V$ in a manner similar to section 5.2 .

$$
U^{\lambda}=U_{\mathfrak{k}_{2}}+\lambda^{-1} U_{\mathfrak{p}_{2}} \text { and } V^{\lambda}=V_{\mathfrak{k}_{2}}+\lambda V_{\mathfrak{p}_{2}} .
$$

Then a family of 1 -forms $\alpha^{\lambda}$ is defined as follows:

$$
\alpha^{\lambda}=\alpha_{\mathfrak{k}_{2}}+\lambda^{-1} \alpha_{\mathfrak{p}_{2}}^{\prime}+\lambda \alpha_{\mathfrak{p}_{2}}^{\prime \prime}=U^{\lambda} d z+V^{\lambda} d \bar{z}
$$


In fact the matrices $U^{\lambda}$ and $V^{\lambda}$ are explicitly given as follows:

$$
U^{\lambda}=\left(\begin{array}{cccc}
\frac{\bar{b}_{z}}{2 \bar{b}} & P & k & \lambda^{-1} b \bar{P} \\
1 & -\frac{\bar{b}_{z}}{2 b} & 0 & k \\
0 & \lambda^{-1} b & \frac{\bar{b}_{z}}{2 b} & P \\
0 & 0 & 1 & -\frac{\bar{b}_{z}}{2 b}
\end{array}\right), \quad V^{\lambda}=\left(\begin{array}{cccc}
\frac{b_{z}}{2 b} & \bar{b} & \bar{P} & \lambda \bar{b} P \\
0 & \frac{b_{z}}{2 b} & \lambda \bar{b} & \bar{P} \\
1 & 0 & -\frac{b_{z}}{2 b} & \bar{k} \\
0 & 1 & 0 & -\frac{b_{z}}{2 b}
\end{array}\right) .
$$

Then the projective minimal surface can be characterized by the harmonicity of the conformal Gauss map [29], [4, Theorem 7], and by a family of flat connections.

Theorem B.3 ([29], [4]). Let $\mathfrak{f}$ be a surface in $\mathbb{R P}^{3}$ and $g_{2}$ the conformal Gauss map defined in (B.1). Moreover, let $\left\{\alpha^{\lambda}\right\}_{\lambda \in \mathbb{S}^{1}}$ be a family of 1 -forms defined in (B.3). Then the following are mutually equivalent:

1. The surface $\mathfrak{f}$ is a projective minimal surface.

2. The conformal Gauss map $g_{2}$ is a conformal harmonic map into $\mathcal{Q}$.

3. $\left\{\alpha^{\lambda}\right\}_{\lambda \in \mathbb{S}^{1}}$ is a family of flat connections on $\mathbb{D} \times G$.

Proof. Let us compute the flatness conditions of $d+\alpha^{\lambda}$, that is, the Maurer-Cartan equation $d \alpha^{\lambda}+\frac{1}{2}\left[\alpha^{\lambda} \wedge \alpha^{\lambda}\right]=0$. It is easy to see that except for the $(1,4)$-entry, the Maurer-Cartan equation is equivalent to (2.6). Moreover, the $\lambda^{-1}$-term and the $\lambda$-term of the $(1,4)$-entry are equivalent to that the first equation and the second equation in (3.1), respectively. Thus the equivalence of (1) and (3) follows.

The equivalence of (2) and (3) follows from Proposition A.2, since the family of 1forms $\alpha^{\lambda}$ is given by the involution $\tau_{2}$ and it defines the semi-Riemannian symmetric space $\mathcal{Q}=\mathrm{SL}_{4} \mathbb{R} / K_{2}$.

Remark B.4. The above theorem implies that if $\mathfrak{f}$ is a projective minimal surface, then there exists a family of projective minimal surface $\mathfrak{f}^{\lambda}\left(\lambda \in \mathbb{S}^{1}\right)$ such that $\left.\mathfrak{f}^{\lambda}\right|_{\lambda=1}=\mathfrak{f}$. Projective minimal surfaces of the family have the same projective metric $8|b|^{2} d z d \bar{z}$ but the different conformal classes of cubic forms $\lambda^{-1} b d z^{3}$.

\section{REFERENCES}

[1] M. Black, Harmonic maps into Homogeneous Spaces, Pitman Research Notes in Mathematics Series 255, 1991.

[2] J. Bolton, F. Pedit, L. M. Woodward, Minimal surfaces and the affine Toda field model, J. Reine Angew. Math. 459 (1995), 119-150.

[3] J. Bolton, L. M. Woodward, Congruence theorems for harmonic maps from a Riemann surface into $\mathbb{C P}^{n}$ and $\mathrm{S}^{n}$, J. London Math. Soc. (2) 45 (1992), 363-376.

[4] F. E. Burstall, U. Hertrich-Jeromin, Harmonic maps in unfashionable geometries, Manuscripta. Math. 108 (2002), 171-189.

[5] F. E. Burstall, F. Pedit, Harmonic maps via Adler-Kostant-Symes theory, in: Harmonic Maps and Integrable Systems (A. P. Fordy, J. C. Wood eds.), Aspects of Mathematics E23 (1994), Vieweg, pp. 221-272.

[6] F. E. Burstall, F. Pedit, Dressing orbits of harmonic maps, Duke Math. J. 80 (1985), 353-382.

[7] D. J. Clarke, Integrability in Submanifold Geometry, Thesis, Univ. Bath., 2012. 
[8] S. S. Chern, J. G. Wolfson, Harmonic maps of the two-sphere into a complex Grassmannian manifolds II, Ann. of Math. (2) 125 (1987), 301-335.

[9] G. Darboux, Leçons sur la théorie générale des surfaces I, 1914, II, 1915, second edition, GauthierVillars.

[10] A. Demoulin, Sur deux transformations des surfaces dont les quadriques de Lie n'ont que deux ou trois points caracteristiques, Bull de l'Acad Belgique 19 (1933), 479 -502, 579-592, 1352-1363.

[11] J. F. Dorfmeister, W. Freyn, S.-P. Kobayashi, E. Wang, Survey on real forms of the complex $A_{2}^{(2)}$-Toda equation and surface theory. Complex Manifolds 6 (2019), 194-227.

[12] J. Dorfmeister, I. McIntosh, F. Pedit, H. Wu, On the meromorphic potential for a harmonic surface in a $k$-symmetric space, Manuscripta Math. 92 (1997) no. 2, 143-152.

[13] F. E. Ferapontov, Integrable systems in projective differential geometry, Kyushu J. Math. 54 (2000), no. $1,183-215$.

[14] H. S. Hu, Darboux transformations of Su-chain, in: Differential Geometry. Proceedings of the Symposium in honor of Professor Su Buchin on his 90th Birthday (C. H. Gu, H. S. Hu, Y. L. Xin eds.), World Scientific, 1993, pp. 325-380.

[15] S.-P. Kobayashi, A loop group method for Demoulin surfaces in the 3-dimensional real projective space, Differential Geom. Appl., 40 (2015), 57-66 (arXiv:1301.6325v2 [math.DG])

[16] E. P. Lane, Projective Differential Geometry of Curves and Surfaces, University of Chicago Press, 1932.

[17] C. LeBrun, L. J. Mason, Nonlinear gravitons, null geodesics, and holomorphic disks, Duke Math. J. 136 (2007), 205-273.

[18] E. Musso, L. Nicolodi, Tableaux over Lie algebras, integrable systems, and classical surface theory, Comm. Anal. Geom. 14 (2006), 475-496.

[19] K. Nomizu, T. Sasaki, Centroaffine immersions of codimension two and projective hypersurface theory, Nagoya Math. J. 132 (1993), 63-90.

[20] K. Nomizu, T. Sasaki, Affine Differential Geometry. Geometry of Affine Immersions, Cambridge Tracts in Mathematics 11 (1994), Cambridge University Press.

[21] C. Pa (Zhengguo Bai), A new definition of the Godeaux sequence of quadrics, Amer. J. Math. 69 (1947), 117-120.

[22] C. Pa (Zhengguo Bai), On the surfaces whose asymptotic curves of one system are projectively equivalent, Univ. Nac. Tucumán Revista A3 (1942), 341-349.

[23] G. Pick, Über affine Geometie IV. Differentialinvarianten der Flächen gegenüber affinen Transformationen, Berichte Verh Ges. Wiss. Leibzig 69 (1917), 107-136.

[24] T. Sasaki, Projective Differential Geometry and Linear Homogenous Differential Equations, Rokko Lectures in Math. 5 (1999), Kobe Univ.

[25] T. Sasaki, Line congruence and transformation of projective surfaces, Kyushu J. Math. 60 (2006), 101-243.

[26] B. Su, On certain periodic sequence of Laplace of period four in ordinary space, Sci. Rep. Tohoku Imperial Univ. 25 (1936), 227-256.

[27] B. Su, On certain couples of closed Laplace sequences of period four in ordinary space, Chinese Math. 5 (1964), 151-174.

[28] B. Su, The growth and development of differential geometry in China. (Japanese) Sūgaku 35 (1983), no. 3, 221-228.

[29] G. Thomsen, Sulle superficie minime proiettive, Annali Mat. 5 (1928), 169-184

[30] E. J. Wilczynski, Projective Differential Geometry of Curves and Ruled Surfaces, Teubner, 1906. 
Institute of Mathematics, University of Tsukuba, Tsukuba, 305-8571, Japan

E-mail address: inoguchi@math.tsukuba.ac.jp

Department of Mathematics, Hokkaido University, Sapporo, 060-0810, Japan

E-mail address: shimpei@math.sci.hokudai.ac.jp 